\title{
Entanglement of strongly interacting low-dimensional fermions in metallic, superfluid and antiferromagnetic insulating systems
}

\author{
V. V. França and K. Capelle \\ Departamento de Física e Informática, Instituto de Física de São Carlos, \\ Universidade de São Paulo, Caixa Postal 369, 13560-970 São Carlos, SP, Brazil
}

(Dated: February 26, 2020)

\begin{abstract}
We calculate the entanglement entropy of strongly correlated low-dimensional fermions in metallic, superfluid and antiferromagnetic insulating phases. The entanglement entropy reflects the degrees of freedom available in each phase for storing and processing information, but is found not to be a state function in the thermodynamic sense. The role of critical points, smooth crossovers and Hilbert space restrictions in shaping the dependence of the entanglement entropy on the system parameters is illustrated for metallic, insulating and superfluid systems. The dependence of the spin susceptibility on entanglement in antiferromagnetic insulators is obtained quantitatively. The opening of spin gaps in antiferromagnetic insulators is associated with enhanced entanglement near quantum critical points.
\end{abstract}

PACS numbers: 03.65.Ud, 03.67.Mn, 71.10.Fd, 71.10.Pm

\section{INTRODUCTION}

Entanglement, one of the most surprising predictions of quantum theory, has recently received much attention in the context of quantum information theory and quantum computation. In quantum information theory, entanglement is identified with nonseparable wave functions, and referred to as "quantum correlation", as opposed to "classical correlation". In quantum many-body physics and quantum chemistry, the expression "correlation" is used in a much more restricted sense, meaning those consequences of the particle-particle interaction that arise beyond the mean-field approximation. Both meanings of correlation are conceptually distinct: A Hartree-Fock wave function (a single determinant), e.g., is entangled in the quantum-information sense, but not correlated in the many-body sense. Even a wave function for perfectly noninteracting and spatially widely separated particles can be entangled (as illustrated by the Einstein-Podolsky-Rosen paradox), whereas correlations in the many-body sense vanish for strictly noninteracting particles.

The present paper investigates correlations in the quantum information sense, in a model in which correlations in the many-body sense are known to be strong: the fermionic Hubbard model. The one-dimensional Hubbard model in an external magnetic field has the Hamiltonian

$$
\begin{array}{r}
\hat{H}=-t \sum_{i, \sigma}\left(c_{i \sigma}^{\dagger} c_{i+1, \sigma}+H . c .\right)+U \sum_{i} c_{i \uparrow}^{\dagger} c_{i \uparrow} c_{i \downarrow}^{\dagger} c_{i \downarrow} \\
-\mu \sum_{i \sigma} c_{i \sigma}^{\dagger} c_{i \sigma}-\frac{h}{2} \sum_{i}\left(c_{i \uparrow}^{\dagger} c_{i \uparrow}-c_{i \downarrow}^{\dagger} c_{i \downarrow}\right),
\end{array}
$$

where $t$ describes hopping between neighbouring sites, $U$

*Electronic address: capelle@if.sc.usp.br is the on-site particle-particle interaction, $h$ is a magnetic field, $\mu$ the chemical potential, and the operators $c_{i \sigma}$ satisfy fermionic commutation relations 11]. This Hamiltonian has a long tradition as archetypical model of strongly interacting electrons in solids [2, 3], comprising metallic and insulating phases at repulsive interactions, and a phase with superconducting correlations at attractive interactions. More recently it has also been used to describe fermionic atoms confined in optical lattices 4, 5, 6]. In the absence of spatial inhomogeneity this model has an exact solution in terms of the Bethe-Ansatz (BA) 2, 3, 7], which reduces the problem of finding the many-body ground state and its energy to solving a set of numerically tractable coupled integral equations.

Differently from the Heisenberg model, which is more widely used in investigations of entanglement, the Hubbard model also accounts for the effect of itineracy of particles and possible superconductivity. What are the effects of such complications on entanglement measures and entanglement witnesses?

Entanglement measures for the Hubbard model were proposed and calculated, e.g., in Refs. 8, 9, 10, 11, 12, 13, 14. Specifically, our work builds on, and extends, that of Refs. [8] and 9] on the local von Neumann entropy [15], or single-site entanglement $\mathcal{E}[8,9,12]$ of the Hubbard model. Gu et al., in Ref. [8], numerically study entanglement on finite Hubbard clusters. For $L=70$ sites, they obtain the entanglement entropy as a function of $U$ precisely at haf filling $(n=1)$, whereas for smaller clusters of up to $L=10$ sites they also obtain results for $n \neq 1$. Larsson and Johannesson [9] consider the opposite limit, $L \rightarrow \infty$, in which they obtain the entanglement entropy of noninteracting particles in a half-filled band $(n=1, U=0)$ and of particles with strongly attractive interaction in a half-filled band $(n=1, U \rightarrow-\infty)$ as a function of magnetic field $h$, and of particles with repulsive interaction in zero magnetic field $(h=0, U>0)$ as a function of chemical potential $\mu$ [16]. The main interest of Refs. 8] and [9] was in the behaviour right at critical 
points, where the entanglement or its derivatives were found to be strongly enhanced.

In the present paper, we use an efficient evaluation of the Bethe-Ansatz integral equations, recently developed in the context of density-functional theory [4, 5, 17, 18, 19], in order to explore the entire $n(\mu)-U-h$ phase diagram, both at and away from critical points 20]. Special cases, known from the earlier work described above, are recovered, and complemented by new information on the behaviour in other regions of the phase diagram. We also evaluate an observable not considered in Refs. [8] and [9], namely the spin susceptibility, known to be an entanglement witness 21, 22, 23]. Our results on the spin susceptibility have a direct bearing on the recent reanalysis 24] of earlier experiments [25] of the entanglement in antiferromagnetic cuprate ladders, and also provide a new perspective on the enigmatic spin gap observed in the normal state of cuprate superconductors.

\section{ENTANGLEMENT ENTROPY ACROSS THE PHASE DIAGRAM}

Starting point of the present analysis is the expression of $\mathcal{E}$ in terms of the particle density (or filling factor) $n=n_{\uparrow}+n_{\downarrow}=\left\langle c_{i \uparrow}^{\dagger} c_{i \uparrow}\right\rangle+\left\langle c_{i \downarrow}^{\dagger} c_{i \downarrow}\right\rangle$, the magnetization (or spin density) $m=\left(n_{\uparrow}-n_{\downarrow}\right) / 2$, and the particle-particle interaction $U$,

$$
\begin{array}{r}
\mathcal{E}\left(n, m, \frac{\partial E}{\partial U}\right)=- \\
-\left(\frac{n}{2}-\frac{\partial e}{\partial U}+m\right) \log _{2}\left[\frac{n}{2}-\frac{\partial e}{\partial U}+m\right] \\
-\left(1-n+\frac{\partial e}{\partial U}-m\right) \log _{2}\left[\frac{n}{2}-\frac{\partial e}{\partial U}-m\right] \\
\log _{2}\left[1-n+\frac{\partial e}{\partial U}\right]-\frac{\partial e}{\partial U} \log _{2}\left[\frac{\partial e}{\partial U}\right]
\end{array}
$$

where $e=E(n, m, U) / L$ is the ground-state energy per site.

Equations equivalent to (2) can be found in Refs. 8, 9], where they are evaluated by approximating the energy derivative $\partial E(n, m, U) / \partial U$ in certain special limits [9], or for systems with a few lattice sites [8], in order to study $\mathcal{E}(n, m, \partial E / \partial U)$ near critical points. Our interest here is in the behaviour of the entanglement entropy also away from critical points and special limits. To this end we must have access to the full function $\mathcal{E}(n, m, \partial E / \partial U)$. Below, we obtain this function from numerical solution of the BA equations for $e(n, m, U)$. In the context of applications of density-functional theory to spatially inhomogeneous Hubbard models [4, 5, 17, 18, 19] we have recently obtained such numerical solutions on a dense mesh of values of $n, m$ and $U$.

Figures 1 and 2 illustrate the resulting dependence of the entanglement entropy on the particle-particle interaction and the particle density. Increase of $|U|$, which makes the wave function more correlated in the manybody sense, decreases $\mathcal{E}$, making it less entangled in the quantum information sense. Conversely, for noninteracting particles $(U=0)$ the entanglement entropy reaches a maximum. This behaviour of $\mathcal{E}(U)$ is due to a nontrivial restriction on the Hilbert space of the one-band Hubbard model, which permits at most one particle of each spin on each site. This restriction remains effective even for $U=0$ (no correlations in the many-body sense) and implies strong correlations (entanglement) in the quantum information sense. We expect that similar Hilbert-space restrictions (e.g., due to conservation laws or topology) in more complex systems lead to other examples of counterintuitive relations between both notions of "correlation".

The qualitative change at the critical points $U=0$ and $n=1$ was predicted in Refs. [8, 9], but Figs. 1 and 2 reveal that even away from critical points the dependence of $\mathcal{E}(n, m, U)$ displays considerable structure. For positive $U$ (repulsive interactions) the entanglement curve as a function of $U$ is almost flat, except at the critical point $n=1$, where a pronounced $U$ dependence is observed, and the curve drops from its theoretical maximum $\mathcal{E}=2$ to below the values observed for $n=0.5$. This different behaviour at $n=1$ reflects the Mott metal-insulator transition. The rapid drop of the entanglement entropy in the insulating phase is due to the freezing of electronic degrees of freedom at $n=1$ and large positive $U$, associated with the transition from the physics of itinerant electrons to that of localized antiferromagnetically coupled spins. At $n \neq 1$ the electrons remain itinerant and the translational degrees of freedom are not frozen out.

We observe in Figs. 1 and 2 that curves corresponding to different values of the system parameters cross, showing that physically distinct states can give rise to the same value of the entanglement entropy, which is thus not a faithfull state function reflecting the microscopic structure of the ground state.

At negative $U$ (attractive interactions) a rapid drop in $\mathcal{E}(U)$ is observed for all fillings. This drop is again due to freezing of degrees of freedom, but at negative $U$ the relevant physics is the crossover from weakly coupled pairs in a BCS-like state to strongly coupled localized dimers in the Bose-Einstein (BE) limit. The number of degrees of freedom is reduced by a factor of 2 in the formation of tightly bound pairs. Accordingly, the numerically determined ratio $\mathcal{E}(U=0) / \mathcal{E}(U \rightarrow-\infty) \approx 2$. The entanglement entropy thus serves as a marker not only for quantum criticality [8, 9, 11, 26] but also for smoother crossovers. The strong dependence of entanglement on the size of the pairing interaction must be taken into account in investigations of entanglement and qubits in the superconducting state [27, 28, 29, 30].

From the point of view of density-functional theory, in particular the Hohenberg-Kohn theorem, the fundamental variables, determining the value of any observable, are the densities $n$ and $m$, and the interaction $U$. From the point of view of experimental manipulation of entangled particles in a device, it is, however, much more convenient to cast the results in terms of variables that are more directly controlled in the laboratory, such as the 


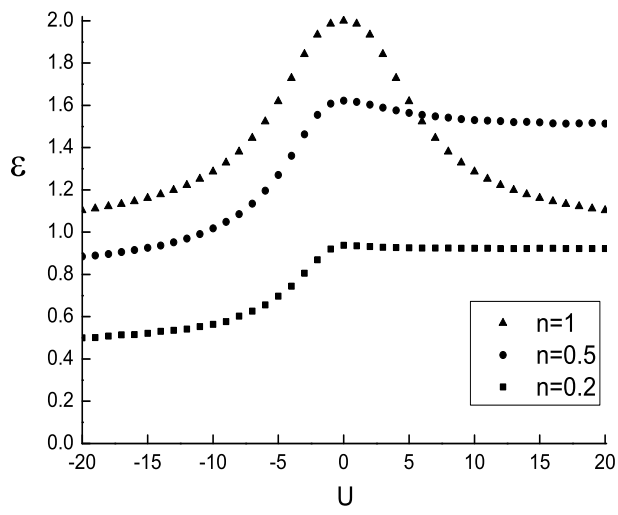

FIG. 1: Local entanglement entropy for $h=0$ as a function of particle-particle interactions, for different particle densities $n$.

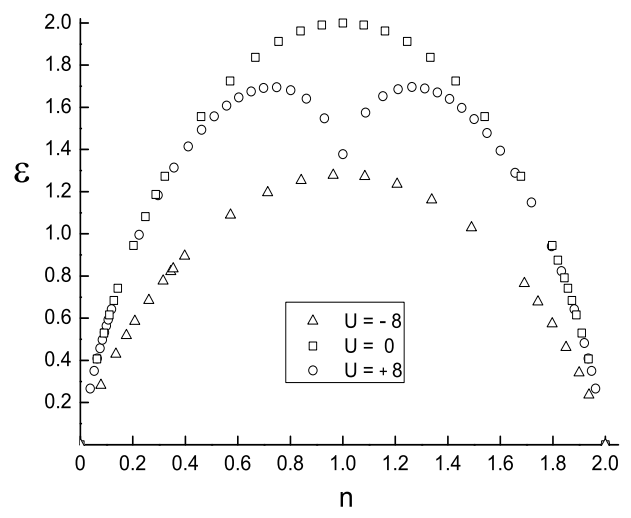

FIG. 2: Local entanglement entropy for $h=0$ as a function of particle density, for different interactions $U$.

applied magnetic field $h$ or the chemical potential $\mu$.

Given the function $\mathcal{E}(n, m, \partial E / \partial U)$, the relations $\mu=$ $\partial E(n, m, U) / \partial n$ and $h=\partial E(n, m, U) / \partial m$ can be used to numerically construct the dual function $\tilde{\mathcal{E}}(\mu, h, U)$. Numerical results for $\tilde{\mathcal{E}}(\mu(n), h=0, U)$ and $\mu(n)$ are plotted in Fig. 3, which shows that the entanglement entropy is a very rapidly varying function of the chemical potential. The behaviour at $U<0$ is similar to that at $U=0$, but for $U>0$ a gap opens at $n=1$ in the energy spectrum, the chemical potential becomes ill-defined, and the entanglement entropy displays a pronounced derivative discontinuity.

Figure 4 illustrates the magnetic-field dependence of the entanglement entropy. For all $U$, the curves terminate at the magnetic field corresponding to magnetic saturation. For $U \geq 0$, the entanglement entropy is maximal at $h=0$ and decreases monotonically with increasing magnetic field, reflecting increased magnetic order. For $U<0$, the entropy only starts to depend on $|h|$ for fields

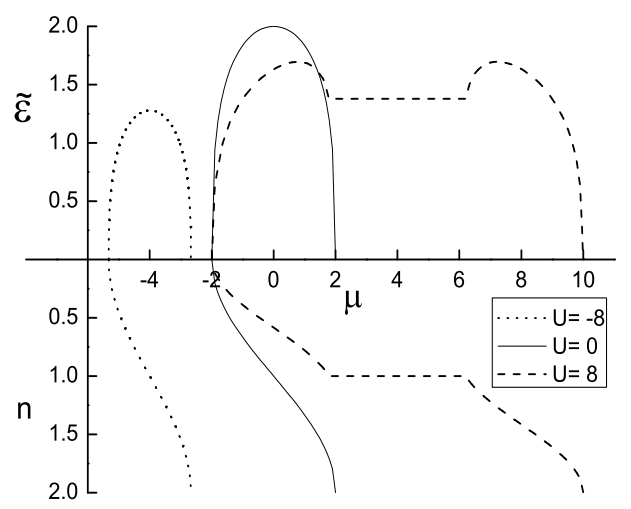

FIG. 3: Local entanglement entropy at $h=0$ as a function of chemical potential (upper panel), and chemical potential as a function of density (lower panel), for different values of the interaction $U$. Note that both panels share a common $\mu$ axis, permiting one to reconstruct the function $\tilde{\mathcal{E}}(n)$.

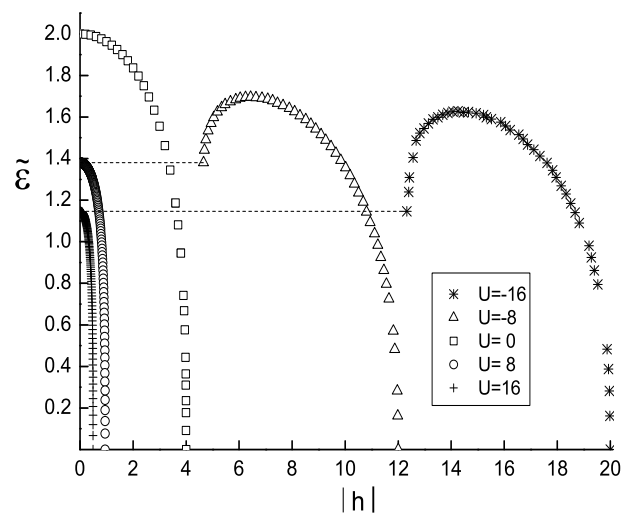

FIG. 4: Local entanglement entropy at $n=1$ as a function of magnetic field $h$ for several values of $U$.

above the spin gap, and displays a round maximum at some $|h|>0$.

\section{SPIN SUSCEPTIBILITY}

As seen above, the behaviour of the entanglement provides direct information about the degrees of freedom available for storing and processing information in systems of strongly interacting fermions. Other observables are also strongly affected by changes in the entanglement. We here focus on the spin susceptibility, (i) because this quantity is an example of an entanglement witness [21, 22, 23], and (ii) because of recent claims 24] that anomalies earlier observed 25] in the spin susceptibility of the antiferromagnetic (AFM) insulator $\mathrm{Cu}\left(\mathrm{NO}_{2}\right)_{2} 2.5 \mathrm{H}_{2} \mathrm{O}$ are due to enhanced entangle- 
ment. This claim is based on the observation that a puzzling and hitherto unexplained 25] drop in the spin susceptibility begins to develop at approximately the same temperature where entanglement is predicted to increase 24.

However, as already indicated in the experimental paper [24], many different explanations for the observed anomaly are conceivable. Moreover, in recent analysis of experiments on $\mathrm{LiHo}_{\mathrm{x}} \mathrm{Y}_{1-\mathrm{x}} \mathrm{F}_{4}$, with predominantly ferromagnetic interactions, precisely the opposite behaviour, namely an increase in the spin susceptibility as entanglement becomes stronger, was argued to occur 31.

It is thus important to verify the connection between the susceptibility and entanglement in a model stripped off all unessential complications, but still complex enough to encompass both antiferromagnetism [24] and entanglement. The spins in $\mathrm{Cu}\left(\mathrm{NO}_{2}\right)_{2} 2.5 \mathrm{H}_{2} \mathrm{O}$ are arranged in chains, so that a description in terms of the onedimensional Hubbard model, which at $n=1$ and $U \rightarrow \infty$ is equivalent to the AFM Heisenberg model with $J=$ $4 t^{2} / U$, becomes possible.

The zero-temperature spin susceptibility of the Hubbard model can be related to the ground-state energy via $\chi^{-1}=\partial^{2} E(n, m, U) /\left.\partial m^{2}\right|_{m=0}$, and may be obtained numerically from the Bethe Ansatz equations [32] as a function of $U$. From Fig. 1 we see that for $U>0$ and $n=1$ the function $\mathcal{E}(U)$ is invertible. Hence, we can numerically construct the function $\chi^{-1}(U(\mathcal{E}))=\chi^{-1}(\mathcal{E})$. For $n=1$ (consistent with the fact that the experiments were done on insulators), this function is plotted in Fig. [5] which clearly shows that the susceptibility decreases with increasing entanglement entropy. Hence, in a minimal (but still realistic) model of antiferromagnetic insulators the susceptibility indeed drops sharply as the system's wave function becomes more entangled. This result strongly suggests that the observation of a drop in the spin susceptibility in $\mathrm{Cu}\left(\mathrm{NO}_{2}\right)_{2} 2.5 \mathrm{H}_{2} \mathrm{O}$ [25] as entanglement grows 24 is not a coincidence, or an artifact.

These Hubbard-model calculations, and according to 24] also the experiments of Ref. [25], show that enhanced entanglement in AFM systems produces a sharp drop in the spin susceptibility. The Hubbard-model calculations reported in the first part of this Letter, as well as related work in [8, 9, 11], show that in the proximity of quantum-critical points the entanglement entropy or its derivatives can be very strongly enhanced. By putting these two pieces of information together we infer that in a strongly entangled state the spin susceptibility of antiferromagnetic systems is suppressed near quantum critical points.

Interestingly, there is another important class of materials that display this type of behaviour, although it is not normally discussed in these terms: The parent compounds of high-temperature superconductors are AFM cuprates, for which much experimental evidence points to the existence of a quantum-critical point. The spin susceptibility of these systems displays a puzzling drop at low temperatures (see, e.g., Fig. 4 and p. 16004 of

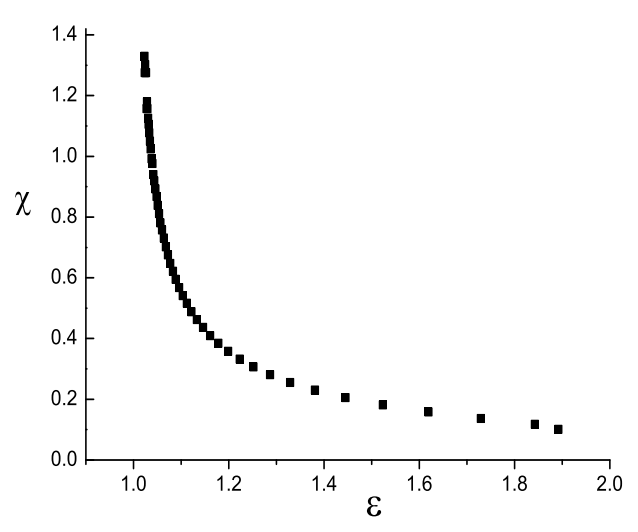

FIG. 5: Spin susceptibility at $n=1$ and $h=0$ as a function of the entanglement entropy.

[33, and the recent re-analysis of those data in Fig. 4 of 34]). This drop is very similar to the one observed in the AFM spin chain $\mathrm{Cu}\left(\mathrm{NO}_{2}\right)_{2} 2.5 \mathrm{H}_{2} \mathrm{O}$, and suggests that the opening of a spin gap in high-temperature superconductors is also be associated with entanglement, persisting at relatively high temperatures.

\section{CONCLUSIONS}

In summary, we have confirmed the previous observation [8, 9, 10, 11, 12] that the entanglement entropy, or its derivatives, are strongly enhanced near quantum critical points. Our Bethe-Ansatz-based treatment additionally reveals that (i) similar behaviour is observed at crossovers, (ii) even away from critical points or crossovers, each physically different phase leaves its distinctive mark on the entanglement entropy, (iii) but in spite of this, the entanglement entropy is not a state function in the thermodynamic sense. It does, however, (iv), provide detailed information about which degrees of freedom are available for storing and processing information in each of the possible phases of the system. (v) Hilbert-space restrictions can lead to counterintuitive relations between the concept of correlation, as employed in many-body physics, and that of entanglement, employed in quantum-information theory.

In addition to the entropy, we also extract another observable closely related to entanglement: the spin susceptibility. We find numerically that in AFM systems enhanced entanglement sharply reduces the spin susceptibility, in agreement with recent reanalysis [24] of earlier experiments 25] but in sharp contrast to what was found in ferromagnetic systems [31]. This observation suggests that the enigmatic spin gap, observed above the critical temperature in cuprate superconductors, may also be a consequence of enhanced entanglement due to proximity of a quantum critical point. Note that high- 
temperature entanglement in cuprates was suggested on different grounds also in 30].

Acknowledgments This work was supported by
FAPESP, CNPq and CAPES. We thank V. L. Campo Jr. for help with the numerical solution of the BA equations.
[1] In this paper all energies $(U, \mu$ etc) are given in multiples of the hopping parameter $t$.

[2] F. H. L. Essler, H. Frahm, F. Göhmann, A. Klümper and V. E. Korepin, The One-Dimensional Hubbard Model (Cambridge University Press, 2005).

[3] P. Schlottmann, Int. J. Mod. Phys. B 11, 355 (1997).

[4] V. L. Campo Jr. and K. Capelle, Phys. Rev. A 72, 061602(R) (2005).

[5] X. Gao, M. Polini, M. P. Tosi, V. L. Campo Jr., K. Capelle and M. Rigol, Phys. Rev. B 73, 165120 (2006).

[6] M. Rigol, Phys. Rev. A 72, 063607 (2005). M. Rigol and A. Muramatsu, Phys. Rev. A 69, 053612 (2004).

[7] E. H. Lieb and F. Y. Wu, Phys. Rev. Lett. 20, 1445 (1968).

[8] S.-J. Gu, S.-S. Deng, Y.-Q. Li and H.-Q. Lin, Phys. Rev. Lett. 93, 086402 (2004).

[9] D. Larsson and H. Johannesson, Phys. Rev. Lett. 95, 196406 (2005). 96, 169906(E) (2006).

[10] D. Larsson and H. Johannesson, Phys. Rev. A 73, 042320 (2006).

[11] L.-A. Wu, M. S. Sarandy, D. A. Lidar and L. J. Sham, eprint quant-ph/0512031

[12] P. Zanardi, Phys. Rev. A 65, 042101 (2002).

[13] A. Anfossi, P. Giorda, A. Montorsi and F. Traversa, Phys. Rev. Lett. 95, 056402 (2005).

[14] V. E. Korepin, Phys. Rev. Lett. 92, 096402 (2004).

[15] C. H. Bennett, H. J. Bernstein, S. Popescu and B. Schumacher, Phys. Rev. A 53, 2046 (1996).

[16] We reproduce those results, as corrected in the erratum to Ref. [9], as limiting cases of our Bethe-Ansatz-based treatment.

[17] N. A. Lima, M. F. Silva, L. N. Oliveira and K. Capelle, Phys. Rev. Lett. 90, 146402 (2003).

[18] N. A. Lima, L. N. Oliveira and K. Capelle, Europhys.
Lett. 60, 601 (2002).

[19] M. F. Silva, N. A. Lima, A. L. Malvezzi and K. Capelle, Phys. Rev. B 71, 125130 (2005).

[20] Density-functional theory allows to treat small clusters and extended systems within the same computational framework, which can be extended to include a wide spectrum of spatial inhomogeneites [4, 5, 17, 18, 19].

[21] M. Wiesniak, V. Vedral and C. Brukner, New J. Phys. 7, 258 (2005).

[22] L.-A. Wu, S. Bandyopadhyay, M. S. Sarandy and D. A. Lidar, Phys. Rev. A 72, 032309 (2005).

[23] O. Gühne and N. Lütkenhaus, Phys. Rev. Lett. 96, 170502 (2006).

[24] C. Brukner, V. Vedral, and A. Zeilinger, Phys. Rev. A 73, 012110 (2006).

[25] L. Berger, S. A. Friedberg and J. T. Schriempf, Phys. Rev. 132, 1057 (1963).

[26] L.-A. Wu, M. S. Sarandy and D. A. Lidar, Phys. Rev. Lett. 93, 250404 (2004).

[27] Y. Nakamura, Y. A. Pashkin and J. S. Tsui, Nature 398, 786 (1999).

[28] J. E. Mooji, T. P. Orlando, L. Levitov, L. Tian, C. H. van der Wal and S. Lloyd, Science 285, 1036 (1999).

[29] P. Zanardi and X. Wang, J. Phys. A 35, 7947 (2002).

[30] V. Vedral, New J. Phys. 6, 102 (2004).

[31] S. Ghosh, T. F. Rosenbaum, G. Aeppli and S. N. Coppersmith, Nature 425, 48 (2003); V. Vedral, ibid , 28 (2003).

[32] H. Shiba, Phys. Rev. B 6, 930 (1972).

[33] T. Nakano, M. Oda, C. Manabe, N. Momono, Y. Miura and M. Ido, Phys. Rev. B 49, 16000 (1994).

[34] P. A. Lee, N. Nagaosa and X.-G. Wen, Rev. Mod. Phys. 78, 17 (2006). 